\title{
Avaliação do Efeito Pró-oxidante no PP, em Blendas com PHB
}

\author{
Paulo A. Santos, Marina N. Oliveira \\ SABIC Innovative Plastics, Campinas, SP \\ Marco-Aurelio De Paoli, Vanessa G. Freitas \\ Universidade Estadual de Campinas, UNICAMP
}

\author{
Derval S. Rosa \\ Universidade Federal do ABC, Santo André, SP, UFABC
}

\begin{abstract}
Resumo: O interesse por polímeros biodegradáveis tem aumentado devido ao baixo impacto ambiental dos mesmos. No entanto, muitos desses produtos disponíveis no mercado são frágeis, difíceis de processar e têm alto custo. Uma das técnicas para superar essas deficiências é através de blenda com outros polímeros. Este trabalho avaliou várias composições de blenda PHB/PP, utilizando-se um aditivo pró-degradante deste último, de forma a tornar a formulação oxibiodegradável em solo simulado (ASTM D6003). O comportamento mecânico, térmico e químico de corpos de prova de tração das amostras enterradas foi acompanhado através de medidas de resistência à tração, DSC, FTIR, GPC e SEM. Parte das amostras foi envelhecida em estufa a $90{ }^{\circ} \mathrm{C}$ por 10 dias, de forma a acelerar a oxidação do PP pelo aditivo. A blenda composta por $56 \mathrm{wt} \%$ de PP / $40 \mathrm{wt} \%$ de PHB / 3 wt $\%$ de GMA / 1 wt $\%$ de MnSt foi a mais susceptível à oxibiodegradação, tendo reduzido depois de 180 dias de enterramento em solo, a tensão de tração em $40 \%$, alongamento na ruptura em $46 \%$ e a massa em $24 \%$. Estes valores foram superiores ao próprio PHB puro e o efeito sinérgico ocorrido nestas blendas foi atribuído à aceleração da degradação do PHB pelo PP oxidado.
\end{abstract}

Palavras-chave: Poli- $\beta$-(hidroxibutirato), polipropileno, blenda, oxibiodegradação, injeção.

\section{Evaluation of the Effect of Pro-degrading Additives in PP, Blended with PHB}

\begin{abstract}
The interest in biodegradable polymers has increased because of their environmental advantages. Blending them with other polymers is one of the techniques currently employed to overcome their brittleness, poor processing and cost. This work evaluated different blend compositions of PHB/PP, using a pro-degrading additive for this latter, to make the formulation prone to oxo-biodegradability in simulated soil (ASTM D6003). The mechanical, thermal and chemical behavior of buried injection molded tensile bars was monitored through measurements of tensile strength, DSC, FTIR, GPC and SEM. Part of the samples was oven aged at $90{ }^{\circ} \mathrm{C}$ for 10 days in order to accelerate the PP oxidation by the additive. The blend made of $56 \mathrm{wt} \%$ of PP / $40 \mathrm{wt} \%$ of PHB / $3 \mathrm{wt} \%$ of GMA / $1 \mathrm{wt} \%$ of MnSt was the most susceptible to oxo-biodegradation, having reduced after 180 days of burring in soil, its tensile stress by $40 \%$, tensile elongation by $46 \%$ and mass by $24 \%$. These values were even higher than the ones for the pure PHB. This synergic effect was attributed to the acceleration of PHB degradation by the oxidized PP.
\end{abstract}

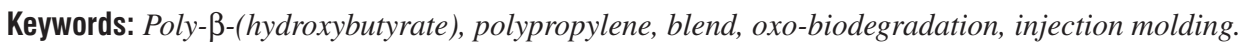

\section{Introdução}

O crescimento do consumo de polímeros foi vertiginoso nas últimas décadas, principalmente movido pelas indústrias de embalagem, construção civil e automotiva. No Brasil o consumo per capita passou de 10 para $30 \mathrm{~kg} /$ habitante-ano em uma década ${ }^{[1]}$. Esse resultado deve-se, basicamente, ao baixo custo de produção desses materiais, sua leveza, elevada resistência mecânica e facilidade de fabricação de peças nas mais variadas formas, tamanhos e cores. A cada ano novos tipos são lançados no mercado, enquanto os já conhecidos se tornam cada vez mais populares.

Uma característica importante dos polímeros é a longevidade e, embora haja muitas iniciativas para sua reciclagem, um enorme volume de embalagens ou peças que já cumpriram seu papel, ainda é descartado todos os dias no meio ambiente onde podem permanecer mais de 100 anos, por serem resistentes ao ataque dos microorganismos.

Nesse contexto, o interesse e o desenvolvimento de resinas biodegradáveis têm crescido muito e gerado grande evolução tecnológica associada. Dentre esses polímeros, destaca-se o poli- $\beta$-(hidroxibutirato), PHB, que pertence à família dos polihidroxialcanoatos (PHA), e é acumulado como reserva energética por muitas bactérias dos gêneros Gram positivas e negativas ${ }^{[2]}$.

Entretanto, o alto custo de produção desses materiais, a baixa disponibilidade de fornecimento e sua limitada processabilidade em comparação aos plásticos convencionais, tem-se constituído em barreiras para expansão de seu consumo. O desenvolvimento de

Autor para correspondência: Paulo A. Santos, SABIC Innovative Plastics, Rua Manoel Thomaz, 545, CEP 13067-230,

Campinas, SP, Brasil, e-mail: paulo.santos@sabic-ip.com 
blendas com outros polímeros, tais como as poliolefinas, é uma maneira de diminuir o custo e melhorar o processamento dos biodegradáveis ${ }^{[3]}$. As poliolefinas porém, como a maioria dos derivados de petróleo, não são biodegradáveis devido à sua alta resistência a microrganismos que, por sua vez, está relacionada com sua hidrofobicidade e alta massa molar, além da falta de grupos funcionais reconhecíveis pelo sistema enzimático microbiano $^{[4]}$.

A incorporação de grupos carbonila na cadeia polimérica, através do emprego de aditivos conhecidos como "pró-oxidantes", é a técnica mais utilizada para facilitar a desintegração das poliolefinas. Na presença de oxigênio, esses aditivos aceleram a degradação do polímero gerando grupos hidroperóxidos, carboxilas e cetonas e provocando a cisão das cadeias macromoleculares. Calor e luz UV são os principais ativadores deste processo ${ }^{[5]}$.

Os plásticos aditivados com esses catalisadores são chamados de "oxi-biodegradáveis" e podem interagir com o ecossistema por meio de duas etapas: abiótica e biótica. A degradação abiótica pode ser controlada pela intensidade da foto e da termo-oxidação e produz fragmentos oxidados com rápida queda da massa molar. Já na etapa biótica, os fragmentos oxidados são passíveis de serem colonizados por microrganismos que utilizam o polímero como substrato ${ }^{[6]}$. No entanto, é bom ressaltar que há ainda razoável controvérsia sobre a ocorrência desta etapa ${ }^{[7]}$.

Este trabalho teve como objetivo melhorar a tenacidade e processabilidade, bem como reduzir o custo de materiais à base de PHB através de blenda com polipropileno, usando aditivos oxidantes para aumentar a degradabilidade deste último, após descarte. No ensaio de biodegradação avaliou-se a perda de massa e de outras propriedades do material, após enterramento em solo simulado, composto com quantidades definidas de areia, terra, húmus e água com temperatura e umidade controladas, conforme ASTM D6003 ${ }^{[8]}$.

\section{Experimental}

\section{Materiais}

PP copolímero com densidade, $\mathrm{d}=0,905 \mathrm{~g} / \mathrm{cm}^{3}$; índice de fluidez, IF $=8 \mathrm{~g} / 10 \mathrm{~min}\left(230{ }^{\circ} \mathrm{C} / 2,16 \mathrm{~kg}\right)$; temperatura de fusão, $\mathrm{T}_{\mathrm{m}}=136{ }^{\circ} \mathrm{C}$. PHB com $\mathrm{d}=1,23 \mathrm{~g} / \mathrm{cm}^{3} ; \mathrm{IF}=28 \mathrm{~g} / 10 \min \left(190{ }^{\circ} \mathrm{C} / 2,16 \mathrm{~kg}\right)$; massa molar, Mw 150 kg/mol. GMA (copolímero de etileno com metacrilato de glicidila, PE- $g$-GMA) com $\mathrm{d}=0,94 \mathrm{~g} / \mathrm{cm}^{3} ; \mathrm{IF}=5 \mathrm{~g} / 10 \mathrm{~min}\left(190^{\circ} \mathrm{C} / 2,16 \mathrm{~kg}\right)$; $\mathrm{T}_{\mathrm{m}}=105^{\circ} \mathrm{C}$. Estearato de manganês, MnSt.

\section{Metodologia}

Para uma primeira análise das propriedades das blendas utilizaram-se diferentes porcentagens de PP e PHB sem ou com o compatibilizante GMA em concentrações de 3 ou 5 wt $\%$ (porcentagem em massa), reduzindo-se o teor de PP proporcionalmente, como mostrado na Tabela 1. Essas misturas foram processadas em extrusora duplarrosca interpenetrante, corrotante Werner \& Pfleiderer (WP-25, Tamm, diâmetro = 25 mm,
Tabela 1. Composições preparadas na primeira etapa do trabalho.

\begin{tabular}{ccc}
\hline sem GMA & 3 wt\% GMA & 5 wt\% GMA \\
\hline PP/PHB (wt\%) & PP/PHB (wt\%) & PP/PHB (wt \%) \\
\hline $100 / 0$ & $97 / 0$ & $95 / 0$ \\
$80 / 20$ & $77 / 20$ & $75 / 20$ \\
$60 / 40$ & $57 / 40$ & $55 / 40$ \\
$40 / 60$ & $37 / 60$ & $35 / 60$ \\
$20 / 80$ & $17 / 80$ & $15 / 80$ \\
$0 / 100$ & $0 / 97$ & $0 / 95$ \\
\hline
\end{tabular}

L/D = 36), de acordo com os seguintes parâmetros de extrusão: perfil de temperatura de 130/ 140/ 150/ $160 / 160{ }^{\circ} \mathrm{C}$ da alimentação até a matriz, com rotação de $280 \mathrm{rpm}$, vazão de $20 \mathrm{~kg} / \mathrm{h}$ e torque de 40 N.m. Os espaguetes foram resfriados em calha de água, secos com ar comprimido e picotados.

$\mathrm{O}$ material granulado foi secado por $3 \mathrm{~h}$ a $80{ }^{\circ} \mathrm{C} \mathrm{e}$ corpos de prova (cp) de tração e impacto Izod, com $3,2 \mathrm{~mm}$ de espessura, foram moldados por injeção utilizando-se equipamento PIC 140 (PIC, Brasil), com os seguintes parâmetros: perfil de temperatura de 140, 150, $160,170{ }^{\circ} \mathrm{C}$ da alimentação até o bico, pressão de injeção de 30 a 40 bar, temperatura do molde de $60{ }^{\circ} \mathrm{C}$ e tempo de resfriamento de $6 \mathrm{~s}$.

Foram realizados ensaios de tração usando-se equipamento de ensaios K2000NP (Kratos, Cotia-SP) e impacto Izod com entalhe à $23{ }^{\circ} \mathrm{C}$, com equipamento M09 (Microtest, Taipei-Taiwan).

Após a definição do melhor teor de compatibilizante (3 wt\%), utilizou-se essa composição como base para analisar o efeito do agente pró-oxidante do PP, o MnSt, na concentração de $1 \mathrm{wt} \%$. As duas melhores blendas desta etapa, juntamente com o PP e PHB foram submetidas ao teste de biodegradação, com o monitoramento da perda de resistência mecânica e de massa em cp de tração. Para tanto, os cp foram enterrados por 180 dias em solo simulado, preparado de acordo com a norma ASTM D6003 ${ }^{[8]}$ e acondicionado em bandejas plásticas armazenadas em prateleiras sem luz e em temperatura de $22 \pm 3{ }^{\circ} \mathrm{C}$. A cada 30 dias, $10 \mathrm{cp}$ eram retirados, limpos, pesados e submetidos ao ensaio de tração.

A composição que apresentou melhor resultado (maior decaimento nas propriedades avaliadas) juntamente com as mencionadas na Tabela 2, foram envelhecidas em estufa por 10 dias à $90^{\circ} \mathrm{C}$, para acelerar a degradação do PP induzida pelo aditivo pró-oxidante. Após este período, as amostras foram pesadas e submetidas à ensaios de tração, para verificar a variação da massa bem como da tensão na força máxima e do alongamento na ruptura, respectivamente.

Depois do envelhecimento em estufa, estes cp foram enterrados em solo simulado por 180 dias. A cada 30 dias, $10 \mathrm{cp}$ eram retirados, limpos, pesados e caracterizados segundo os ensaios abaixo:

a) calorimetria diferencial de varredura (DSC): foi realizada usando-se equipamento Thermal Analyser 2100 (TA Instruments, New Castle-EUA) sob atmosfera de nitrogênio com dois ciclos de aquecimento para se eliminar a história térmica 
Tabela 2. Composição das amostras submetidas ao envelhecimento em estufa.

\begin{tabular}{lcccc}
\hline \multirow{2}{*}{ Sigla } & \multicolumn{4}{c}{ Composição } \\
\cline { 2 - 5 } & PP & PHB & GMA & MnSt \\
\cline { 2 - 5 } & $(\mathbf{w t} \%)$ & $(\mathbf{w t} \%)$ & $\mathbf{( w t \% )}$ & $\mathbf{( w t \% )}$ \\
\hline PP 100 & 100 & - & - & - \\
PP 96 & 96 & - & 3 & 1 \\
$57 / 40$ & 57 & 40 & 3 & - \\
$56 / 40 / \mathrm{Mn} / \mathrm{St}$ & 56 & 40 & 3 & 1 \\
PHB 96 & - & 96 & 3 & 1 \\
PHB 100 & - & 100 & - & - \\
\hline
\end{tabular}

das amostras. A temperatura de fusão cristalina foi determinada e o grau de cristalinidade calculado pelo valor da entalpia de fusão $(\Delta \mathrm{H})$ do PP e do PHB, utilizando-se os valores de $\Delta \mathrm{H}$ de $209 \mathrm{~J} / \mathrm{g}$ para o $\mathrm{PP}$ e de $146 \mathrm{~J} / \mathrm{g}$ para o PHB, referentes a $100 \%$ de cristalinidade destes polímeros.

b) espectroscopia vibracional na região do infravermelho por reflexão total atenuada (FTIRATR): foi utilizado equipamento Nicolet Nexus 470 (Thermo Fischer Scientific, Waltham-EUA) com intervalo de varredura de $1 \mathrm{~cm}^{-1}$ na faixa de 400 $4000 \mathrm{~cm}^{-1}$. A região da carbonila (1700-1750 $\left.\mathrm{cm}^{-1}\right)$ foi monitorada para se avaliar a degradação do PP e, para o PHB, observou-se o aparecimento de uma banda próxima a $1650 \mathrm{~cm}^{-1}$, referente à formação da ligação $\mathrm{C}=\mathrm{C}$ atribuída à sua degradação ${ }^{[9]}$.

c) cromatografia por permeação em gel (GPC): utilizou-se equipamento VARIAN 9002-SDS (Agilent, Santa Clara-EUA) equipado com injetor Rheodyne, modelo 7125, detector de índice de refração, modelo Star 9040 e integrador VARIAN 4400. Numa primeira etapa o PHB foi extraído da blenda com clorofórmio a frio e esta solução injetada no GPC, para se obter sua massa molar $(\mathrm{Mw})$. O PP remanescente na amostra foi dissolvido em triclorobenzeno a $140{ }^{\circ} \mathrm{C}$, que também foi injetado no GPC para determinação de sua massa molar.

d) microscopia eletrônica de varredura (MEV): as amostras previamente submetidas à fratura criogênica em nitrogênio líquido e, posteriormente revestidas com ouro e paládio $(\mathrm{Au} / \mathrm{Pd})$, à $5 \mathrm{kV}$ e corrente de $40 \mathrm{~mA}$ por $60 \mathrm{~s}$, foram observadas com equipamento JSM-6360LV (Jeol, Tokyo-Japão).

\section{Resultados e Discussão}

\section{Propriedades das blendas PP/PHB de diferentes concentrações, sem ou com GMA}

Os resultados dos testes de tensão na força máxima, módulo de elasticidade, alongamento na ruptura em tração e impacto Izod com entalhe, das blendas PP/ PHB/3 wt\% GMA (que apresentou melhor relação custo/ benefício), são apresentados na Tabela 3. Por esses dados, observa-se que à medida que se aumenta a concentração do PP, há uma redução da tensão na força máxima e do módulo elástico mas, por outro lado, um aumento do alongamento na ruptura e da resistência ao impacto Izod.
Tabela 3. Resultados dos testes mecânicos das blendas PP/PHB com 3 wt $\%$ de GMA.

\begin{tabular}{ccccc}
\hline $\begin{array}{c}\text { PP/PHB } \\
(\mathbf{w t} \%)\end{array}$ & $\begin{array}{c}\sigma_{\text {máx. }} \\
(\mathbf{M P a})\end{array}$ & $\mathbf{E}(\mathbf{M P a})$ & $\varepsilon_{\mathbf{b}}(\%)$ & $\begin{array}{c}\text { Impacto } \\
\mathbf{I z o d}(\mathbf{J} / \mathbf{m})\end{array}$ \\
\hline $97 / 0$ & $22,1( \pm 0,3)$ & $776( \pm 38)$ & $407( \pm 19)$ & $89( \pm 9)$ \\
$77 / 20$ & $22,8( \pm 0,6)$ & $1155( \pm 23)$ & $26( \pm 2)$ & $60( \pm 5)$ \\
$57 / 40$ & $25,6( \pm 0,4)$ & $1580( \pm 65)$ & $14,1( \pm 0,6)$ & $32( \pm 3)$ \\
$37 / 60$ & $25,4( \pm 0,3)$ & $2217( \pm 154)$ & $11,3( \pm 0,4)$ & $19( \pm 2)$ \\
$17 / 80$ & $27,9( \pm 0,2)$ & $2766( \pm 141)$ & $9,2( \pm 0,7)$ & $17( \pm 1)$ \\
$0 / 97$ & $30,1( \pm 0,8)$ & $3520( \pm 160)$ & $8,9( \pm 0,5)$ & $23( \pm 2)$ \\
\hline
\end{tabular}

Esta era uma característica esperada, já que a adição do PP foi feita, justamente, com o propósito de aumentar a tenacidade do $\mathrm{PHB}^{[10]}$.

\section{Propriedades mecânicas das blendas PP/PHB/GMA com MnSt}

Os resultados dos testes de tensão na força máxima, módulo de elasticidade, alongamento na ruptura em tração e impacto Izod com entalhe das blendas PP/PHB, contendo $3 \mathrm{wt} \%$ de GMA e $1 \mathrm{wt} \%$ do aditivo pró-oxidante MnSt, são mostrados na Tabela 4. Comparando-se esta com a Tabela 3 é possível verificar, que a adição do agente pró-oxidante provoca pequena variação nas propriedades mecânicas tendendo ligeiramente, a aumentar a tensão na força máxima e o módulo elástico e diminuir o alongamento na ruptura e a resistência ao impacto Izod. Isto pode ser explicado por esse aditivo já causar certa degradação (e, com isto, possível aumento na cristalinidade) do PP durante o processamento por extrusão e injeção, reduzindo sua massa molar e, consequentemente, sua tenacidade ${ }^{[10]}$. Pelo método ponderado da multiplicação dos valores obtidos (metodogia 6 sigma-SABIC) identificaram-se as duas blendas de melhor balanço de propriedades (valores superiores da última coluna da Tabela 4), que foram utilizadas na etapa seguinte.

\section{Biodegradação das blendas PP/PHB 56/40 e $36 / 60$ wt\% com GMA e pró-oxidante}

Os gráficos da Figura 1 mostram a variação da tensão na força máxima e do alongamento na ruptura em tração, em função do período de enterramento para as duas melhores blendas anteriores, comparando-as com as sem MnSt, juntamente com o PP e o PHB individuais com 3 wt $\%$ de GMA e 1 wt $\%$ de MnSt. A Figura 2 mostra o mesmo para a perda de massa. Nestes gráficos de tendência é possível observar que a blenda 56/40/MnSt foi a mais susceptível à biodegradação, apresentando após 180 dias de enterramento, a maior perda de tensão, alongamento e de massa, com reduções de $\sim 35,17$ e $16 \%$, respectivamente.

\section{Avaliação das propriedades das blendas PP/PHB/GMA/MnSt pós-estufa}

As amostras PP 96 e 56/40/MnSt envelhecidas em estufa e enterradas em solo simulado por 180 dias apresentaram queda nas propriedades bem superior à 
Tabela 4. Resultados dos testes mecânicos das blendas PP/PHB/3 wt\% GMA/1 wt\% MnSt.

\begin{tabular}{cccccc}
\hline $\mathbf{P P} / \mathbf{P H B}(\mathbf{w t} \%)$ & $\sigma_{\text {máx. }}(\mathbf{M P a})$ & $\mathbf{E}(\mathbf{M P a})$ & $\varepsilon_{\mathbf{b}}(\boldsymbol{\%})$ & Impacto Izod $(\mathbf{J} / \mathbf{m})$ & Multiplicação $(\times \mathbf{1 0})$ \\
\hline 96/0/MnSt & $24,4( \pm 0,4)$ & $906( \pm 33)$ & $385( \pm 18)$ & $95( \pm 7)$ & \\
76/20/MnSt & $23,6( \pm 0,5)$ & $1323( \pm 25)$ & $13,5( \pm 0,9)$ & $33( \pm 6)$ & 139 \\
$56 / 40 / \mathrm{MnSt}$ & $26,2( \pm 0,5)$ & $1781( \pm 75)$ & $11,8( \pm 0,4)$ & $35( \pm 3)$ & 194 \\
$36 / 60 / \mathrm{MnSt}$ & $26,3( \pm 0,8)$ & $2545( \pm 147)$ & $9,6( \pm 0,7)$ & $24( \pm 4)$ & 154 \\
16/80/MnSt & $32,1( \pm 0,2)$ & $3126( \pm 151)$ & $7,4( \pm 0,5)$ & $16( \pm 2)$ & 120 \\
$0 / 96 / \mathrm{MnSt}$ & $31,3( \pm 0,9)$ & $3580( \pm 168)$ & $8,8( \pm 0,5)$ & $24( \pm 1)$ & \\
\hline
\end{tabular}

Tendência

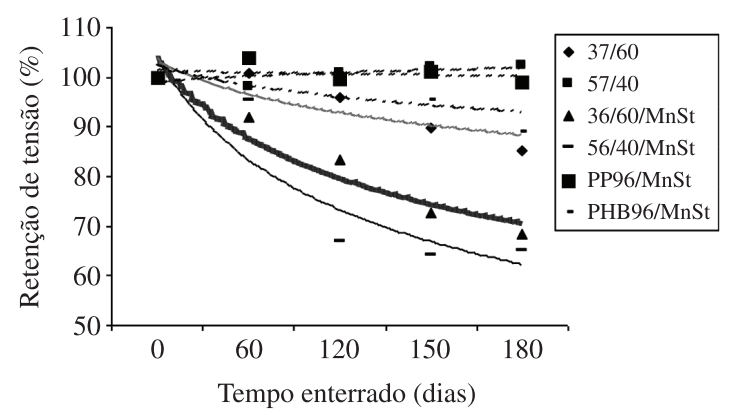

(a)

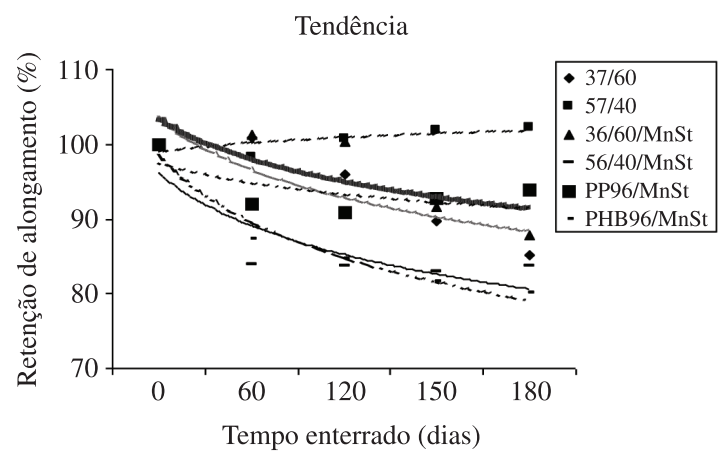

(b)

Figura 1. Variação relativa da tensão na força máxima (a) e do alongamento à ruptura (b) em tração em função do tempo de enterramento em solo simulado.

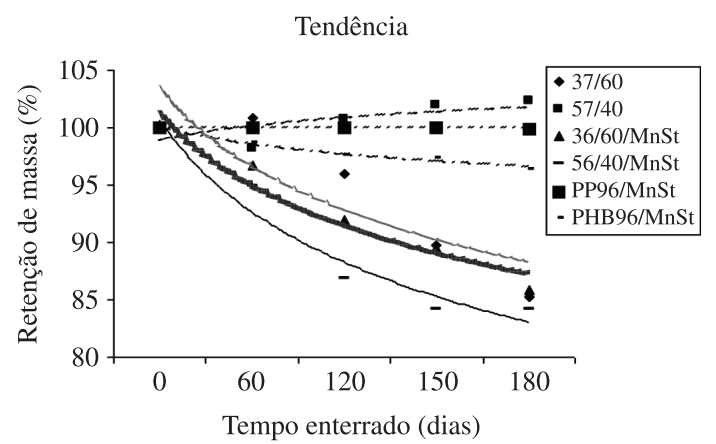

Figura 2. Variação relativa da massa em função do tempo de enterramento em solo simulado.

obtida na etapa anterior, comprovando o efeito catalítico do calor sobre a ação do aditivo pró-oxidante ${ }^{[4]}$. Para o PP 96 as reduções foram de $\sim 50 \%$ na tensão na força máxima, $\sim 95 \%$ no alongamento à ruptura e $\sim 16 \%$ na massa, enquanto para a blenda as reduções foram de $\sim 40 \%, \sim 46 \%$ e $\sim 24 \%$, respectivamente, nas mesmas propriedades.

\section{Análise por FTIR-ATR}

Para o PP 100, PHB 100, PHB 96 e a blenda 57/40 não se notou nenhuma diferença significativa entre os espectros destes materiais virgens e os obtidos após tratamento em estufa ou enterramento em solo. Deste modo pode-se concluir que, praticamente não houve degradação destas amostras após envelhecimento, detectável por esta técnica.

A Figura 3 mostra uma comparação dos espectros (deslocados no eixo y) dos materiais virgens, após 10 dias na estufa e com 90 e 180 dias de enterramento em solo simulado. Quando se compara os espectros do PP 96 virgem com o que permaneceu 10 dias em estufa, observa-se o aparecimento de uma banda larga e assimétrica, com máximo próximo a $1720 \mathrm{~cm}^{-1}$, que é atribuída à ligação $\mathrm{C}=\mathrm{O}$ (carbonila) de formação de um éster ou outros produtos de oxidação ${ }^{[5]}$. Isto indica que houve degradação do PP pela ação do agente oxidante após o período em estufa. Com o enterramento em solo essa banda vai diminuindo e, segundo os mecanismos de biodegradação propostos para o PP na literatura, uma oxidação abiótica prévia é necessária, gerando grupos carbonila que são, posteriormente, metabolizados pelos microorganismos $^{[6]}$.

Comparando-se o espectro da blenda 56/40/MnSt virgem com a que permaneceu por 10 dias em estufa, observa-se o aumento da banda próxima a $1720 \mathrm{~cm}^{-1}$ atribuída à ligação $\mathrm{C}=\mathrm{O}$, o que indica o início da degradação do PP devido ao agente pró-oxidante. Já quando se compara com os espectros das amostras que foram enterradas em solo, observa-se uma mudança acentuada nas suas bandas características, com a diminuição do pico em $1720 \mathrm{~cm}^{-1}$ e o aparecimento de uma banda em $1650 \mathrm{~cm}^{-1}$, esta atribuída à formação da ligação $\mathrm{C}=\mathrm{C}$, referente à degradação do $\mathrm{PHB}^{[9]}$. Estes dados evidenciam que está havendo degradação simultânea do PHB e do PP nesta blenda com o tempo de enterramento, possivelmente devido à ação microbiana do solo, sendo no caso deste último, iniciada nos seus fragmentos oxidados ${ }^{[6]}$.

\section{Análise por DSC}

Os resultados da análise por DSC com dois ciclos de aquecimento são mostrados na Tabela 5. Através deles, é possível verificar que não houve alterações significativas 


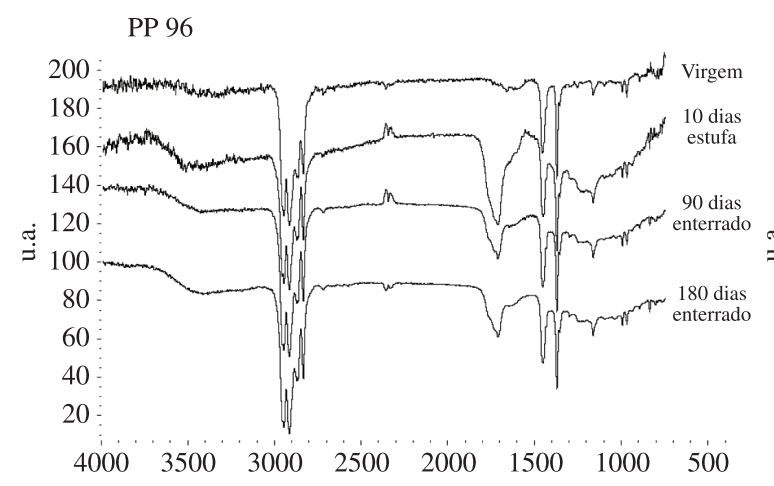

Wavenumbers $\left(\mathrm{cm}^{-1}\right)$

(a)

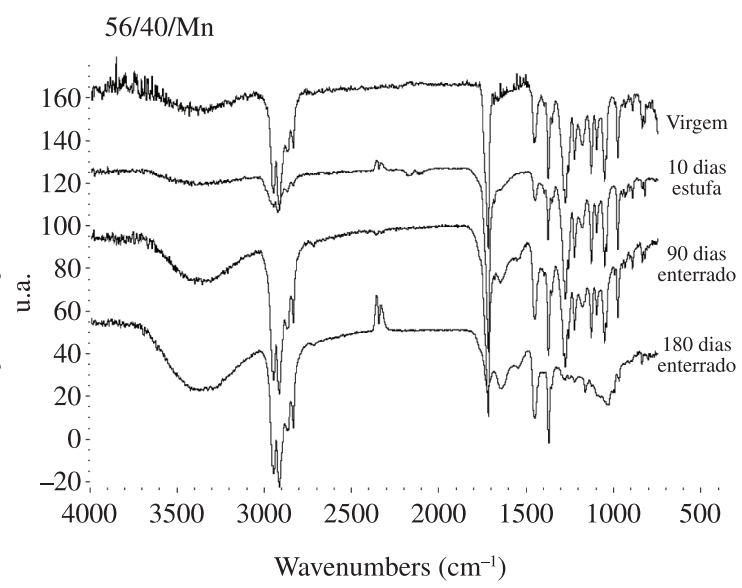

(b)

Figura 3. Espectros de FTIR-ATR para o PP 96 (a) e blenda 56/40/MnSt (b) virgens, após estufa e após enterramento em solo simulado.

Tabela 5. Dados obtidos por DSC das blendas e dos polímeros individuais.

\begin{tabular}{|c|c|c|c|c|c|c|c|}
\hline \multicolumn{2}{|c|}{ Amostra } & \multicolumn{3}{|c|}{ Virgem } & \multicolumn{3}{|c|}{180 dias } \\
\hline & & $\mathbf{T}_{\mathrm{m}}\left({ }^{\circ} \mathbf{C}\right)$ & $\Delta\left(\mathbf{J ~ g ~}^{-1}\right)$ & Cristalinidade (\%) & $\mathbf{T}_{\mathrm{m}}\left({ }^{\circ} \mathbf{C}\right)$ & $\Delta \mathrm{H}\left(\mathbf{J ~ g ~ g}^{-1}\right)$ & Cristalinidade (\%) \\
\hline \multicolumn{2}{|l|}{ PP 100} & 135 & 54,90 & 27 & 135 & 55,64 & 27 \\
\hline \multicolumn{2}{|l|}{ PP 96} & 135 & 64,90 & 31 & 126 & 75,07 & 36 \\
\hline \multicolumn{2}{|l|}{ PHB 100} & 173 & 78,12 & 54 & 171 & 83,37 & 57 \\
\hline \multicolumn{2}{|l|}{ PHB 96} & 172 & 82,85 & 57 & 172 & 88,31 & 60 \\
\hline \multirow[t]{2}{*}{$57 / 40$} & $\mathrm{PP}$ & 134 & 28,94 & 14 & 135 & 27,19 & 13 \\
\hline & PHB & 175 & 27,16 & 18 & 175 & 29,66 & 20 \\
\hline \multirow[t]{2}{*}{$56 / 40 / \mathrm{MnSt}$} & $\mathrm{PP}$ & 136 & 4,35 & 2 & 134 & 19,59 & 10 \\
\hline & PHB & 170 & 27,41 & 19 & 161 & 8,15 & 6 \\
\hline
\end{tabular}

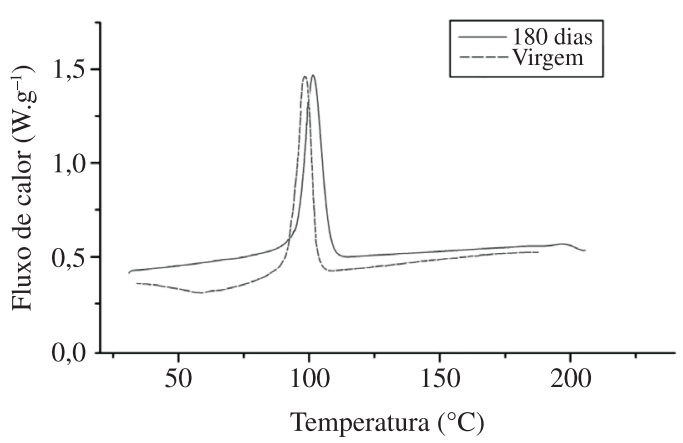

Figura 4. Curvas de DSC de resfriamento da blenda 56/40/MnSt virgem e 180 dias enterrada em solo simulado.

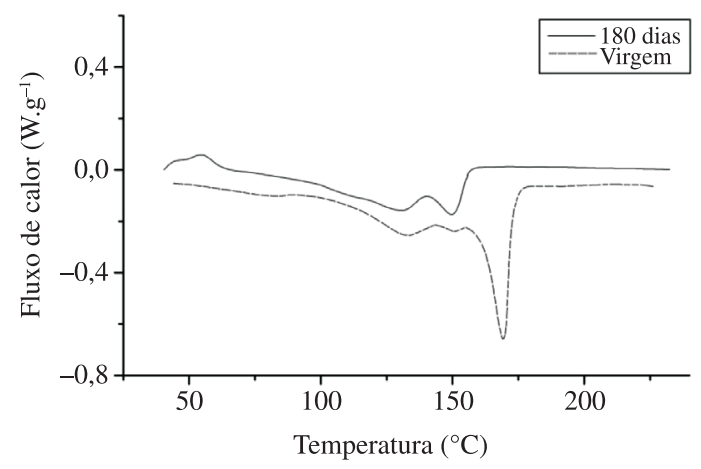

Figura 5. Curvas de DSC de aquecimento da blenda 56/40/MnSt virgem e 180 dias enterrada em solo simulado.
Tabela 6. Variação da massa molar $(\overline{\mathrm{Mw}})$ por GPC com o tempo de enterramento para o PP e PHB, nas composições estudadas.

\begin{tabular}{|c|c|c|c|c|}
\hline \multirow[t]{2}{*}{ Amostra } & \multicolumn{2}{|c|}{$\mathrm{PP}\left(\times 10^{3} \mathrm{~g} / \mathrm{mol}\right)$} & \multicolumn{2}{|c|}{ PHB $\left(\times 10^{3} \mathrm{~g} / \mathrm{mol}\right)$} \\
\hline & PP 96 & $56 / 40 / \mathrm{MnSt}$ & РНB 96 & $56 / 40 / \mathrm{MnSt}$ \\
\hline virgem & 345,2 & 339,6 & 149,1 & 150,3 \\
\hline 10 dias de estufa & 298,1 & 286,7 & 148,6 & 148,1 \\
\hline 30 dias em solo & 273,0 & 293,9 & 146,4 & 130,3 \\
\hline 60 dias em solo & 249,6 & 281,9 & 137,1 & 111,8 \\
\hline 90 dias em solo & 278,5 & 272,7 & 131,3 & 94,2 \\
\hline 120 dias em solo & 291,1 & 287,1 & 141,8 & 78,7 \\
\hline 150 dias em solo & 305,6 & 279,6 & 138,7 & 64,5 \\
\hline 180 dias em solo & 280,2 & 265,9 & 135,8 & 47,5 \\
\hline
\end{tabular}

tanto na temperatura de fusão, quanto no grau de cristalinidade das amostras PP 100, PHB 100, PHB 96 e 57/40, o que condiz com os resultados anteriores de FTIR-ATR.

Para o PP 96 observou-se uma diminuição da $T_{m}$ e um aumento de sua cristalinidade com o tempo de enterramento, atribuídos à sua degradação. A queda na temperatura de fusão pode ser explicada pelo fato das moléculas do polímero degradado possuírem menor massa molar, provavelmente formando lamelas cristalinas menos espessas, que necessitam de uma menor energia para se desfazerem ${ }^{[11,12]}$. Já o aumento da cristalinidade pode ser devido a uma maior mobilidade dessas moléculas mais curtas, que resulta em menor 
enovelamento dos segmentos localizados na fase amorfa e, por conseguinte, permitem que a cristalização prossiga até um nível maior ${ }^{[4,13]}$.

Na blenda 56/40/MnSt (Figuras 4 e 5) observou-se o mesmo efeito do PP mencionado acima, com redução da $\mathrm{T}_{\mathrm{m}}$ e aumento de sua cristalinidade. Para o PHB, porém, embora tenha havido também uma diminuição da $\mathrm{T}_{\mathrm{m}}$, ocorreu uma redução de sua cristalinidade. Isto foi confirmado por outros autores que atribuem esse comportamento à ocorrência da ação enzimática nas duas

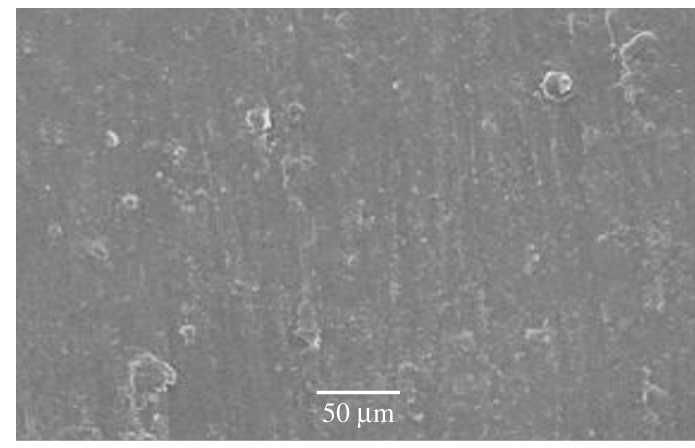

(a)

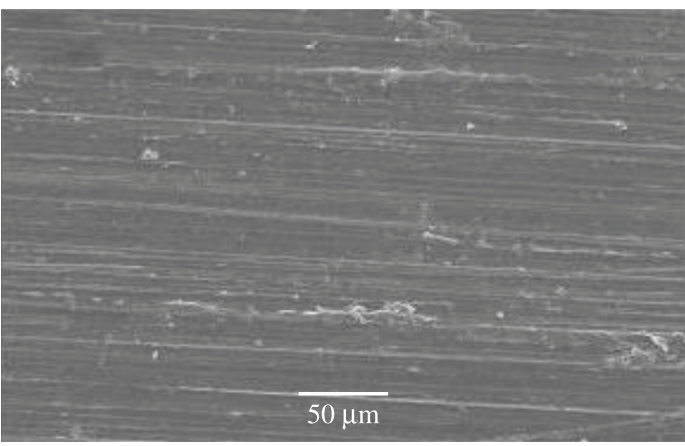

(c)

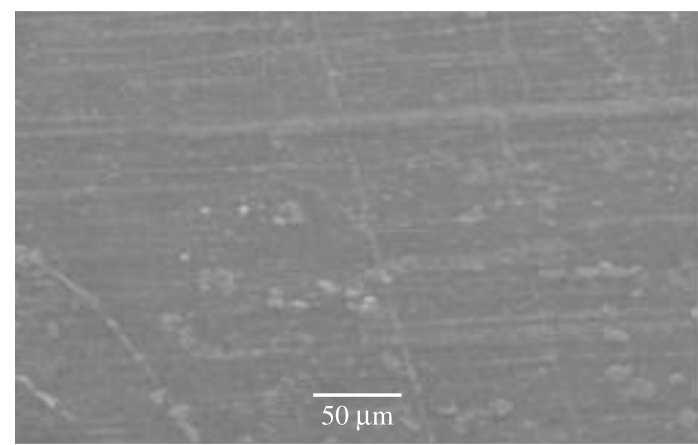

(e)

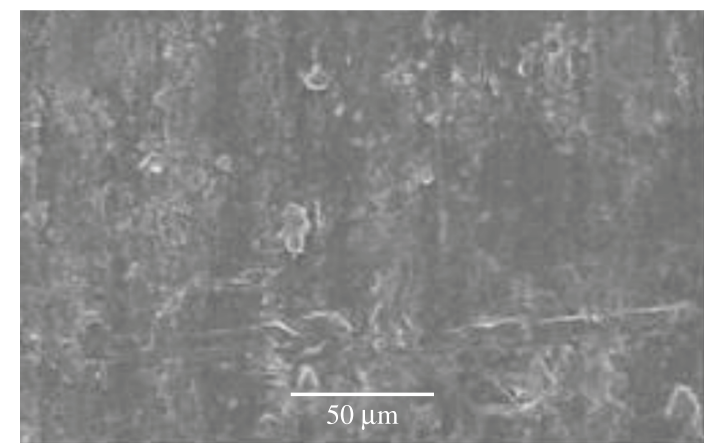

(g)

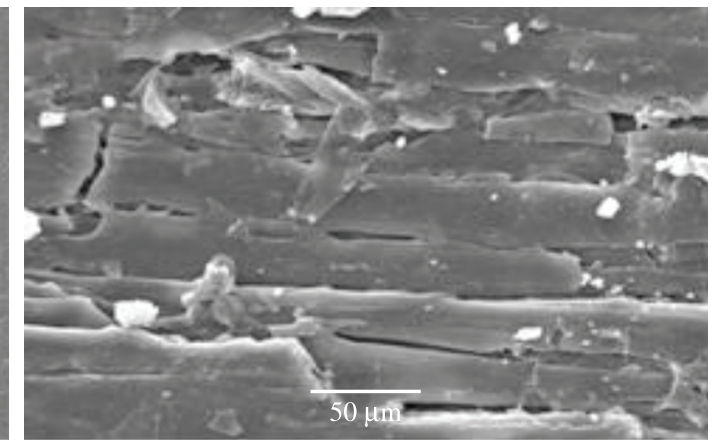

(b)

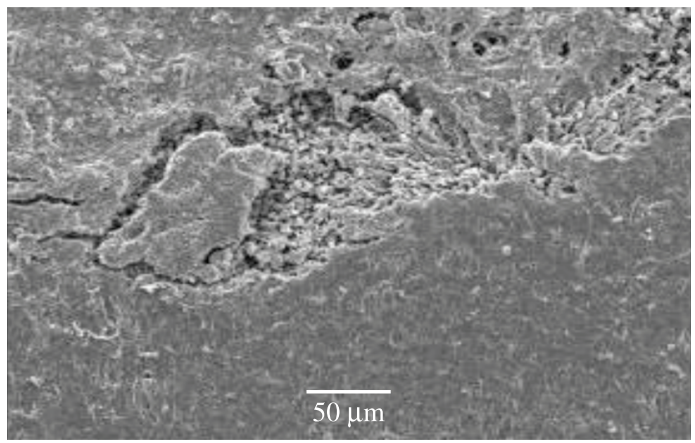

(d)

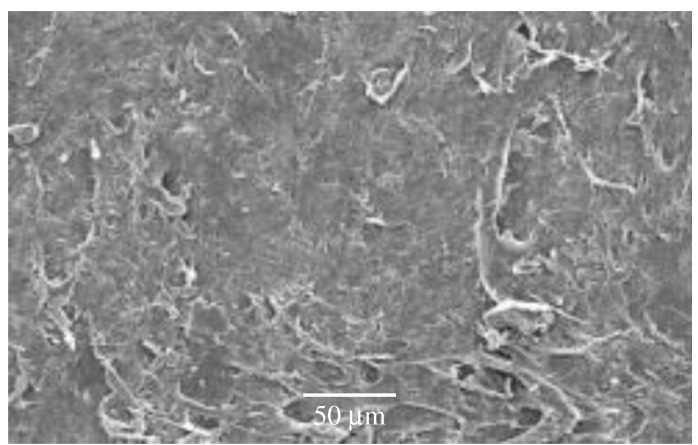

(f)

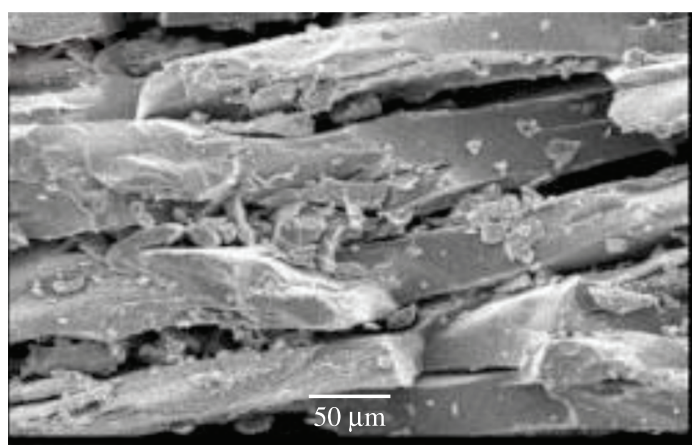

(h)

Figura 6. Micrografias obtidas por SEM das amostras virgens e após 180 dias em solo de: PP 96 (a, b); PHB 96 (c, d); PP/PHB 57/40 (e, f); PP/PHB 56/40/MnSt (g, h). A barra de escala corresponde a $50 \mu$. 
fases, sendo que, inicialmente há o ataque na fase amorfa e, depois, na cristalina, principalmente em seus finais de cadeia ${ }^{[4,14]}$. Uma outra possível explicação é que a degradação do PHB tenha sido tão acentuada que, devido a grande diminuição da sua massa molar, a formação de cristais foi dificultada, por não conseguir gerar muitos núcleos estáveis.

Outro ponto importante a destacar é a menor cristalinidade do PP e PHB nesta blenda virgem ( 2 e $\sim 19 \%$, respectivamente), que também favorece e acelera sua biodegradação ${ }^{[14]}$.

\section{Análise por GPC}

Os resultados da análise de variação da massa molar $(\overline{\mathrm{Mw}})$ por GPC mostrados na Tabela 6, evidenciam a degradação do PP e do PHB nas amostras, indicada pela diminuição de suas massas molares com o tempo de enterramento em solo, após envelhecimento em estufa. Essa redução na $\overline{\mathrm{Mw}}$ foi maior para o PHB na blenda 56/40/MnSt, com $~ 68 \%$, seguida do PP na mesma blenda, com $22 \%$ e pelo PP 96, com 19\%. A massa do PHB 96 caiu somente $9 \%$, o que prova o efeito sinérgico de biodegradação ocorrido com a blenda 56/40/MnSt.

Esse comportamento está, provavelmente, ligado à influência da degradação do PP sobre o PHB, fazendo com que este último, sofra um processo mais intenso de biodegradação na presença da poliolefina oxidada. Uma possível explicação seria que a degradação preferencial do PP pelo pró-oxidante o torna hidrofílico e gera poros na sua superfície, facilitando a adsorção e permeação da umidade e favorecendo a hidrólise do PHB que tem, assim, sua biodegradação também acelerada ${ }^{[15]}$. Por outro lado, embora essa forte redução da massa molar do PP cause sua fragmentação, no período de enterramento empregado neste estudo, talvez ela não tenha atingido ainda o valor mínimo necessário para torná-lo totalmente biodegradável ${ }^{[7]}$.

\section{Microscopia Eletrônica de Varredura}

A morfologia superficial dos cp antes e depois do enterramento foi analisada por SEM (Figura 6). Nas amostras virgens de PP 96, observou-se uma superfície homogênea, lisa e contínua. Já nos cp enterrados, são evidenciados fortes sinais de degradação, constatados pela presença de trincas e poros na superfície, agora rugosa. Também nota-se um esbranquiçamento acentuado, que pode ser atribuído ao aumento do grau de cristalinidade na superfície do polímero ${ }^{[13]}$.

O PHB 96 virgem apresenta morfologia superficial homogênea, compacta e contínua, enquanto na amostra enterrada em solo observa-se uma alta densidade de poros, oriundos da degradação sofrida pelo material. Para a blenda 57/40 virgem, nota-se uma boa compatibilidade e o cp apresenta superfície homogênea e compacta. Já na amostra enterrada em solo, são observados alguns pequenos poros que sugere a ocorrência do início de uma degradação superficial, atribuída à fase PHB, uma vez que o PP sem pró-oxidante não sofre ação dos microrganismos.

Para a blenda 56/40/MnSt virgem observa-se uma separação de fases, atribuída à adição do agente pró-oxidante, possivelmente associada com a redução da massa molar e alterações nas regiões cristalinas, que produz blendas menos compatíveis ${ }^{[16]}$. Enquanto o cp virgem apresenta também, uma superfície compacta e homogênea, no enterrado em solo nota-se a presença de várias estrias e trincas, que devem estar ligadas à ocorrência de uma pronunciada degradação iniciada na superfície, causando erosão, porosidade e redução nas propriedades mecânicas ${ }^{[17]}$. Pelas análises anteriores (FTIR-ATR, DSC, GPC, propriedades mecânicas, perda de massa), conclui-se que ambas as fases (PP e PHB) foram degradadas, favorecidas por sua incompatibilidade causada pelo pró-oxidante ${ }^{[18]}$. Essa degradação se inicia nas interfaces da blenda heterogênea, que é considerada parte importante do processo biodegradativo, por facilitar a penetração da umidade e dos microorganismos ${ }^{[1,12]}$.

\section{Conclusão}

Através de blenda com PP, utilizando o GMA como agente compatibilizante, foi possível melhorar a tenacidade do PHB, avaliada por ensaios de impacto Izod e alongamento na ruptura em tração e a adição de $3 \mathrm{wt} \%$ deste aditivo foi suficiente para otimizar essas propriedades mecânicas. À medida que se aumentou a concentração do PP na blenda, houve uma redução da tensão na força máxima e do módulo elástico em tração, mas por outro lado, um aumento do alongamento na ruptura e da resistência ao impacto Izod.

A adição do agente pró-oxidante, $\mathrm{MnSt}$, provocou pequena variação nas propriedades mecânicas tendendo ligeiramente, a aumentar a tensão na força máxima e o módulo elástico em tração e diminuir o alongamento na ruptura e a resistência ao impacto Izod, devido à degradação (e, com isto, possível aumento da cristalinidade) do PP já provocada pelo aditivo durante o processamento.

No teste de biodegradação em solo simulado, verificou-se que a blenda $56 \mathrm{wt} \%$ de PP, $40 \mathrm{wt} \%$ de PHB, $3 \mathrm{wt} \%$ de GMA e $1 \mathrm{wt} \%$ de MnSt foi a mais susceptível, mesmo comparada ao PHB puro. Com o envelhecimento em estufa, a ação do pró-oxidante acelerada pelo calor foi evidenciada pela maior queda nas propriedades após nova biodegradação, chegando a $46 \%$ no alongamento à ruptura e $24 \%$ na massa.

$\mathrm{O}$ efeito sinérgico ocorrido nessa blenda pode ser atribuído à degradação preferencial do PP pelo pró-oxidante, que o torna hidrofílico e gera poros na superfície da amostra, facilitando a adsorção e permeação da umidade e consequente hidrólise do PHB. Este fato foi corroborado pelas análises de FTIR-ATR, DSC, GPC e SEM.

Embora esses resultados se mostrem promissores quanto à biodegradabilidade de blendas PP/PHB e tenham gerado o pedido de uma patente internacional (WO2009/133516A1), sugere-se que mais testes sejam efetuados, principalmente, com relação a outras normas como a ASTM D6400 e D5988, para avaliar os gases liberados durante a biodegradação e se o material foi completamente bioassimilado pelo solo. 


\section{Agradecimentos}

MAP agradece ao CNPq (bolsa de pesquisador IA) e à FAPESP (Proc. 2010/17804-7).

\section{Referências Bibliográficas}

1. Associação Brasileira da Indústria do Plástico. - "Anuário da ABIPLAST" (2010). Disponível em: <www.abiplast. com.br>. Acesso em: 10 jun. 2011.

2. Sudesh, K.; Abe, H. \& Doi Prog, T. - Polym. Sci., 25, p.1503 (2000).

3. Scott, G.; Lemaire, J.; Jakubowicz, I.; Ojeda, T. \& Hebbar, P. - "OPA Responds to attack from Hydro-bio Industry", Ox.biodeg. Plastics Association. Disponível em: <http:// www.biodeg.org>. Acesso em: 18 ago. 2010.

4. Martelli, S. M.; Fernandes, E. G. \& Chiellini, E. - J. Therm. Anal. Calorim., 97, p.853 (2009). http://dx.doi. org/10.1007/s10973-009-0355-2

5. Khabbaz, F. \& Albertsson, A. C. J. - J. Appl. Polym. Sci., 79, p.2309 (2001). http://dx.doi.org/10.1002/10974628(20010321)79:12<2309::AID-APP1038>3.0.CO;2-E

6. Billingham, N. C.; Chiellini, E.; Corti, A.; Baciu, R. \& Wiles, D. - "Environmentally degradable plastics based on oxo-biodegradation of conventional polyolefins", in: Chiellini, E. \& Solaro, R., Plenum Publisher, New York, Biodegrad. polymer and Plastics, p.313 (2003).

7. Ammala, A.; Bateman, S.; Dean, K.; Petinakis, E.; Sangwan, P.; Wong, S.; Yuan, Q.; Long; Yu; Patrick, C. \& Leong, K. H. - Prog. Polym. Sci., 36, p.1015 (2011). http:// dx.doi.org/10.1016/j.progpolymsci.2010.12.002

8. American Society for Testing and Materials. - "Determining Weight Loss From Plastic Materials Exposed to Simulated Municipal Solid-Waste (MSW) Aerobic Compost Environment: ASTM D 6003-96", Annual Book of ASTM Standards, 09.04 (1996).
9. Aoyagi, Y.; Yamashita, K. \& Doi, Y. - Polym. Degrad. Stab., 76, p.53 (2002). http://dx.doi.org/10.1016/S01413910(01)00265-8

10. Sadi, R.; Fechine, G. J. M. \& Demarquette, N. R. - "Optimal Processing PP/PHB Blends", in: 23rd Polym Proces Society Meeting, Salvador (2007).

11. Campos, A.; Marconato, J. C. \& Franchetti, S. M. M. - Polímeros, 20, p.295 (2010). http://dx.doi.org/10.1590/ S0104-14282010005000039

12. Gonçalves, S. P. C.; Chinaglia, D. L. \& Martins-Franchetti, S. M. J. - J. Polym. Environ., 17, p.280 (2009). http:// dx.doi.org/10.1007/s10924-009-0150-y

13. Vanin, M.; Santana, C.C.; Torriani, I. L.; Privelic, T. \& Duek, E. A. R. - Polímeros., 14, p.187 (2004). http://dx.doi. org/10.1590/S0104-14282004000300015

14. Sadi, R. K.; Fechine, G. J. M. \& Demarquette, N. R. - "Macro2010", in: 43rd IUPAC World Polymer Congress, Glasgow (2010).

15. Lucas, N.; Bienaime, C.; Belloy, C.; Queneudec, M.; Silvestre, F. \& Nava-Saucedo, J. E. - Chemosphere, 73, p.429 (2008). PMid:18723204. http://dx.doi.org/10.1016/j. chemosphere.2008.06.064

16. Rosa, D. S.; Grillo, D.; Bardi, M. A. G.; Calil, M.R.; Guedes, C. G. F., Ramires, E. C. \& Frollini, E. - Polym. Test., 28, p.836 (2009). http://dx.doi.org/10.1016/j. polymertesting.2009.07.006

17. Pachekoski, W. M.; Agnelli, J. A. M. \& Belem, L. P. - Mat. Res., 12, p.159 (2009). http://dx.doi.org/10.1590/S151614392009000200008

18. Na, Y. H.; He, Y.; Nishiwaki, T.; Inagawa, Y.; Osanai, Y.; Matsumura, S.; Saito, T.; Doi, Y. \& Inoue, Y. - Polym. Degrad. Stab., 79, p.535 (2003). http://dx.doi.org/10.1016/ S0141-3910(02)00371-3
Enviado: 01/07/11 Reenviado: $22 / 06 / 12$ Aceito: $26 / 07 / 12$ 